\title{
From Social Requirements to Technical Solutions - Bridging the Gap with User-Oriented Data Security ${ }^{*}$
}

\author{
Ulrich Kohl \\ University of Freiburg \\ Institute for Informatics and Society \\ Telematics Department \\ Friedrichstrasse 50 \\ 79098 Freiburg, Germany \\ kohl@iig.uni-freiburg.de
}

\begin{abstract}
Centralized IT security approaches focus on securing the system against intrusions from the outside, following a centrally administered security policy, in whose definition the end users do not participate. This fact involves two major disadvantages: their own security requirements - which in combination result in the social requirements - are not respected, and their knowledge of the sensitivity of and the need-to-work with the data they own, i.e. produce, store, communicate, modify, and delete, is not taken into account. These disadvantages lead to rather static security mechanisms growing more and more ineffective in fulfilling the users' and societal security requirements.

To eliminate these disadvantages, the integration of the end users as active participants in the security domain is needed. This means that they have to get a means to communicate all their security requirements to the IT system in a convenient way.

This article describes an object-oriented approach called user-oriented data security and its realization which allows the end users to specify their own needs and thus adds an element of decentralization to centralized security. A decentralized approach enables the end user to specify his security needs and, at the same time, to take responsibility for them.

The concept of user-oriented data security has been elaborated in the SaferCom project, a joint project of the Telematics Department of the Institute for Informatics and Society at the University of Freiburg, Germany, the Clinical Computing Center, also in Freiburg, Germany, and the IBM European Networking Center, in Heidelberg, Germany. In the SaferCom project a prototype for secure handling of open distributed documents was developed and is demonstrated in a health-care-application with patient data.
\end{abstract}

\section{Introduction}

Society's requirements for security and privacy in communication systems can be found

* I am happy to acknowledge IBM Germany's contribution to this work. The SaferCom-Project has been partially funded by IBM Germany under the project number S141. 
throughout the history of mankind. Since the beginning of telecommunication - the first systems in form of courier posts in $2900 \mathrm{BC}$ - appropriate security mechanisms for the protection of the communication were developed, beginning with the couriers carrying homing pigeons as a form of negative acknowledgement signals [Holz94]. Since then, telecommunication techniques and security threats and requirements have increased dramatically, having reached a temporary peak with the upcome of the information age with its possibilities of unlimited global telecommunication for each individual. Modern security mechanisms, e.g. cipher mechanisms, access control lists and matrices, or protocols for key exchange and authentication, can be designed practically secure.

Today's security requirements, however, cannot be described merely in specifying requirements for the basic security mechanisms. Modern society has abstract requirements which can be derived from common sense or of its expression in existing laws. In an age when secure technical systems can be developed, the problem of security is drifting from technological security, whose main interest lies in the sound calculation and possible certainty about the nonhuman world, towards an area of human relationships which are technologically induced, so that the aim of security is becoming once again a state between humans. This fact finds its expression in the list of the following non-technical criteria for information security:

- Informational self-determination: The need for individuals to be in full control over which persons or organizations have access to personal data on them.

- Non-repudiation: The impossibility to negate having issued a data operation; this concerns the operation as well as the content of the data created or communicated.

- Reproduceability: The understandability of actions and reactions in the system.

- Design potential: The ability of end users to design their information and communication devices, services, and interfaces according their needs.

These requirements address the needs of locally distributed end users. Satisfying the sum of their independant individual, decentralized requirements promises to result in a high security standard..

The basic security technology yet exists, but its applications must be presented to the end user in a different manner, making him an integral part of the security domain and hence filling in the gap between the technical security tools themselves and their control by the end user. The integration of the end user is also considered by the U.S. National Research Council in [NRC91]. The concept of user-oriented security aims at solving this problem.

This paper is organized in six sections. Section 2 will describe which security mechanisms and security services exist today and how their use leads to a gap between their service and the end user requirements. Section 3 introduces a new, object-oriented approach called user-oriented data security bridging this gap. In section 4 , the requirements of hospital information systems and a specific health care application will be presented. Section 5 comes with the description of our prototype's technical architecture, and section 6 will close with a short summary.

\section{State of the art in security and security mechanisms}

\subsection{Approaches to security}

The wide dissemination of information and communication systems also leads to their use 
for applications in handling sensitive data in different public areas. The first efforts to prevent abuses and theft were made by systems manufacturers who developed mechanims to defend the systems' owners against the users or third parties. Of course, this may be meaningful in preventing fraud or espionage, and also human error. On the other hand security also has to protect the user from the provider, especially in distributed environments, e.g. when a private user is paying to use a public telecommunications network. A synthesis of the two approaches lies in the integration of the end user in the system security domain, which additionally opens his knowledge about security needs to the system. Suggestions for this so-called multilateral security [Rann93] can be found in actual proposals for security evaluation criteria, for example in the ISO's Evalution Criteria for IT Security [ISO94] under the headings unobservability, anonymity, unlinkability, pseudonymity, non-repudiation, and connection integrity.

Furthermore, centralized security systems which secure the systems against their users tend to detain the users from their work instead of supporting them. This tendency may also be seen in the context of rigid data protection laws which do the same thing on a more general level, namely forbid the use of information and communication technology in cases where misuses of data could be possible.

Pure decentralization, i.e. complete control of security by the end users, will not be the solution. We see user-oriented security as an add-on to the usual methods which have to be used to specify a framework in which end users have some freedom to define their own needs. Within the framework adherence to laws could be maintained. As we will see, the possibility of delegating responsibility to the end user could be one reason for making laws less restrictive, allowing for greater accessibility to IT technology in organizations.

Although, for achieving all these advantages, the end users need the possibility to communicate their needs in a natural and easy way.

\subsection{Security mechanisms today}

Powerful technical security mechanisms which could be used to implement user-oriented data security already exist. Computer science can build a toolbox to construct more complex mechanisms and services including the services an end user needs.

Figure 1 shows a set of basic security services [Mart92] which are built out of the security mechanisms. These services serve no special purpose but can be used solely or in combination to render a higher level of service to the end user. The general-purpose list of basic security services is identification, authentication, authorization, access control, integrity, non-repudiation, and audit service.

For the provision of these services many security mechanisms have been developed and are subject to standardization. An overview of the services, the mechanisms and the objects they operate on is given in [Denn82] or [DaPr89], for example. 


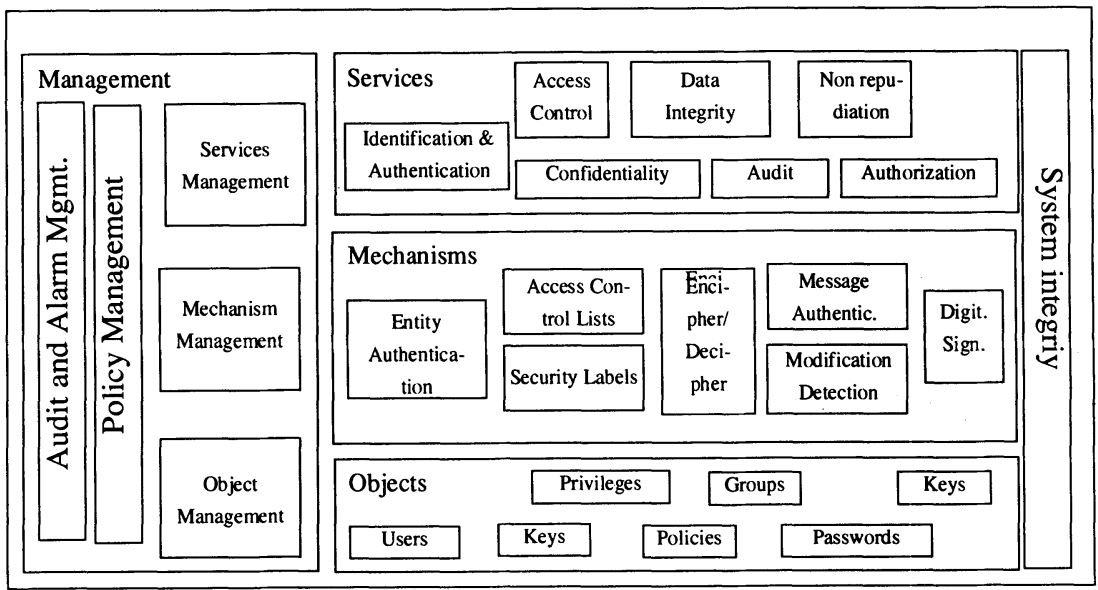

Figure 1: Security Mechanism Toolbox

\subsection{The gap between user requirements and security mechanisms}

When communicating directly, humans have implicit control of their communication including the security characteristics. Complex requirements can be met and controlled quite naturally. In oral communication, one may for example whisper to communicate in a confident manner. The invention of writing changed communication and its security issues for the first time. New mechanisms - seals, secret writing, etc. - have been introduced and established in culture during centuries. Technical communication changed human communication in a similar way the second time. Although some security mechanisms could be adopted and new ones were developed, the problem remains that the user lost control over his communication characteristics. To solve this problem, the users have to be given a means to communicate their requirements to the technical communication system. Like in natural communication, this is a form of meta-communication which underlies the semiotic rules. The machine has to be able to understand the pragmatics the user has [Wall92].

The gap between the mechanisms and the way an end user expects to use them has to be bridged in several steps. Complex security services can be built from basic ones, and the end users' complex requirements (at the pragmatic level) can be decomposed into simple ones (at the semantical and syntactical levels). At the user interface, the requirements are mapped into the appropriate combination of basic security services. Figure 2 illustrates this scheme. 


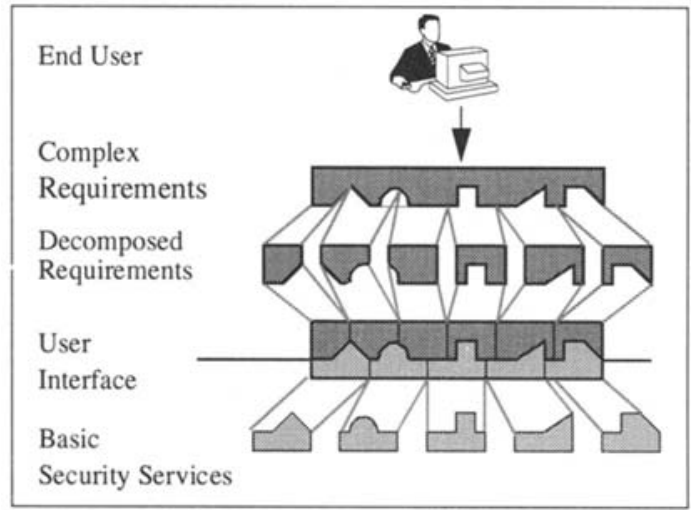

Figure 2: From complex user requirements to Basic Security Services

\section{Bridging the gap with user-oriented security}

For the appropriate presentation of the end users' possibilities to specify security characteristics, both sides - the end user's view and needs and the way a security service can be provided - have to be modeled to facilitate the mapping. For this description, an object-oriented method was chosen.

\subsection{The Object-Oriented Approach}

Our new approach introduces a decentralized component into the conventional mechanisms. As described above, integrating the end users implies using their knowledge of their security needs. This allows for more dynamics which can be more easily adapted to specific situations than would be possible using only centralized, off-line security services. The users are free to act, but the responsibility is also delegated to them.

From the end user's abstract point of view, data processing and communication in organisations consist of creating, storing, manipulating, communicating and deleting information. The rights and duties of users concerning actions and information and the quality of service of the system are subject to the security issues.

Going into more detail and using object-oriented terminology, the universe of users and data consists of subjects and objects. Subjects represent users, but also agents which act on behalf of users. Objects represent the information. Hierarchies exist for both subjects and objects as well as properties and attributes can be defined which can in turn be inherited to superobjects or subobjects. Subjects may also be allowed to allocate properties to subjects in other hierarchy subtrees. Object classes are comparable to data types, whereas subject classes can be derived from job descriptions. When taking distributed systems into account, both subjects and objects may be located on different systems. The overall aim is to solve the paradox of guaranteeing security and privacy in open systems. 


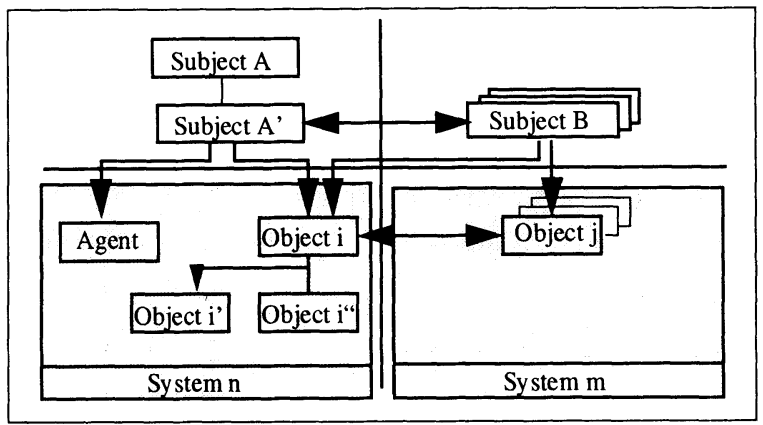

Figure 3: Object-oriented view of data processing

The basic question for security in this world can be reformulated as which subject is allowed to or must perform which operations on which object when with which consequences? It is obvious that general answers cannot be given in a permanently changing environment. The answers to this question, however, vary and must be given on demand by the users during their work, which implies a transition of security aspects from system orientation to user orientation.

\subsection{Modelling with the object-oriented approach}

An interface where users may specify their requirements has to offer high granularity in specification details in conjunction with a powerful aggregation potential. First, the basic elements of the object-oriented model, i.e. the subjects, objects, classes, and the operations need to be defined. Using this model, all sorts of relations, dependencies, rights and duties between users and data can be expressed.

The classification of subjects is relatively easy. As mentioned above, the users of the system are subjects. A user may have rights and duties which are bound to his personal identity, but a more common case will involve the user as belonging to a class and inheriting the predefined rights of that class. The security relevant attributes of subjects are called Privilege Attributes and include the clearance level, the capabilities or the possible roles of this subject class. User classes are called roles [Lawr93]. Roles facilitate the modelling of the security policy to a considerable extent as they decouple the bonding from rights to natural persons. In dynamic environments where users change their duties regularly, roles can be defined according to job descriptions. When persons enter a new job, they will be assigned a new role in which they inherit all their necessary rights.

The classification of the objects depends on the application. In a document archive such as we use for our prototype described below, the objects are hierarchically structured documents where the tree consists of documents and folders. The security relevant properties of object classes are stored in Control Attributes such as access control lists and confidentiality or integrity classes.

The operations also depend on the application. Besides the functional operations of the application, e.g. operations to manipulate documents (a typical list of operations could be create, read, write, append, delete, transfer), parameters for rights or duties can also be defined. These meta-rights may involve the transfer of owned rights or rights to do security manage- 
ment. They have to be specified using several basic services, whereas the specification of security requirements concerning the normal work with the objects is mapped solely on the specification of the access control basic security service. In addition to the operation itself, a behavioral norm must be specified. The distinction between right and duty is established in the literature, but more sophisticated distinctions can be found as on [Lubi93] the four norm list of permission, freedom, duty and prohibition, where permission is the right to execute an operation, freedom is the right to decline to execute an operation, and duty and prohibition express the duty to do or explicitly not to do something.

A complete classification of all requirements for the basic security services cannot be given in this paper, and thus we confine ourselves to a short overview, especially for access control which is the main service used during normal operation. Requirements for the other services are often requirements on the quality of service, e.g. which identification method has to be chosen, if weak or strong authentication is desired etc.

Requirements on access control were discussed above with reference to the question of : which subject is allowed to or must perform which operations on which object when with which consequences? We see that at least a subject or role, an object or object class, an access method and a behavioral norm is necessary to specify a requirement. In addition, requirements on actions, conditions, time, location, hardware, software, context, states of subjects or objects, frequency, urgency, etc. may be specified. All requirements can be expressed following the scheme $(P, S, N, O p, O, A)$ where $P$ is a predicate evaluated before a subject $S$ behaves with the norm $\mathrm{N}$ with using the operation $\mathrm{Op}$ on object $\mathrm{O}$, causing action $\mathrm{A}$ to be taken. The object-oriented approach allows for aggregating basic subject, operation, and object classes so that also complex requirements can be specified.

Using a specification scheme like this, both requirements of a central security policy like During his working hours a physician allowed to read all of his patient data or In an emergency any physician is allowed to read any patient's data, but his actions have to be audited and the security administrator has to be notified, but al so individual requirements like I give the on duty radiologist the nonrecurring right to create a new subdocument in my patient's file containing his diagnosis can be specified. While the central security policy is invariant and is entered and maintained by qualified personnel, the decentral, dynamic specification issued by the end users must take place via their user interface.

\section{Use in a hospital information system}

\subsection{Use of of IT in health care environments}

Use of information and communication technology in a health care environment has many facets. On the one hand, technical progress has made innovative methods available for the creation and management of patient data, e.g. Magnetic Resonance Tomographs (MRT) or Picture Archiving and Communication Systems (PACS) which offer new possibilities for health care. These techniques not only increase the quality of treatment, but also have an impact on the cost.

On the other hand, medical data are considered to be among the most sensitive data for civil use as they contain very detailed, personal information about patients and their health. For centuries, the hippocratic oath has expressed the physicians' duty to respect patients' privacy. Today, this is no longer sufficient and is extended by civil law [DeFa91]. In Germany, the federal data protection law [BDSG91], social law and hospital laws of the German states are 
focusing on this fact. Not only is the privacy of data required, but also the document status of patient data has to be protected as well. Data must not be modified in any way and must not be deleted for a specific period. For example, $X$-ray images have to be archived for 30 years.

The dilemma between the benefits and pitfalls of information technology is clearly visible. It can be used for better and cheaper treatment, the primary goal, but the health data may also be misused. The sensitivity of medical data and its characteristic feature that it can save lives make it hard to determine an appropriate security policy for all situations. Security, however, is not simply a matter of the letter of the law; it is subject to interpretation and in some cases a trade-off [Wäch94] between the two aims has to be made. Patients want their health data kept secret, but security is no longer that important if a patient is in a critical situation for health or life and needs help as soon as possible.

The use of IT in hospital environments is, as has been said, mandatory, although very high security standards are expected [Seel91]. These requirements on hospital information systems not only arise from the security aspect, but also from the purely functional aspect. A large university hospital can provide patients with optimal support through its complex organization, consisting of several clinics, institutes, central labs, and administration which all have to exchange data, but each of them has specific rights and duties which may even be competitive. The organisational entities are often located all over a town, there are even cases in which the radiological clinic itself is distributed, as there may be a radiological department in each distinct clinic. The overall organization is a mix between centralization and decentralization. Patient data are created at different places and at different times, but in order to be a valuable source of information they always have to be complete. We see again the strong need for distributed control of security in such a complex IT system.

\subsection{Requirements of the prototype application}

For our prototype, we studied a very common application, namely the management and communication of patient information. Patient data are multimedia data containing text, audio, graphics and video picture sequences. Most of the data are very sensitive and have the status of documents. Proof of authorship of a document must be documented, not deniable and involves special rights, such as copyright or originator rights.

The end users are the the hospital staff: doctors, nursing, pharmacy and management staff, and al so patients who have the right to take a look at their data. As it cannot be defined in detail in advance who needs to know specific data, the patient's physician has to decide dynamically to whom he gives additional access rights [Sand89]. He is fully responsible for these actions. Delegations of rights and responsibilities also may be transitive.

The security requirements of this kind of application are multilevel. The storage and communication of patient data has to correspond with the security policy and applicable law without detaining the work flow. The information logistics must have the following features:

- The author of any particular piece of information is considered to be its responsible owner.

- Rights and duties on information are not static, but change depending on their context. The context also changes with time, controlled by events. For example, an X-ray image may be altered by the diagnosing radiologist, but after its release to the patient data archive it has the status of a document.

- Rights and duties depend on roles and documents.

- Rights and responsibilities can be delegated and given away. Thus, the problem of security 
and correct use must be coordinated between the distributed users.

- The assignment and delegation of rights must be understandable, i.e. who transferred what to whom when in which context of which rights. [JKP94]

\section{Technical architecture of the prototype}

In order to achieve openness and interoperability, the prototype of the project has been set up considering international standards both for its operation and security. The ISO/OSI Layer 7 standard DFR (Document Filing and Retrieval) [ISO91a] [ISO91b] was chosen to specify the open document archive. DFR offers the service to store, manage and manipulate open distributed documents. Although DFR does not care about security issues, the protocol and the document structure have already been prepared for the integration of other elements.

Security standards have been chosen from the European Computer Manufacturers Association (ECMA) in their standards ECMA TR/46: Security in Open Systems, A Security Framework [ECMA88] and ECMA-138: Security in Open Systems, Data Elements and Service Definitions [ECMA89]. Based on the ISO/OSI security architecture for the Basic Reference Model [ISO89], they provide a model for handling security relevant information and a set of functions to implement some security services using this information. Access protocols and transfer syntax for security information are provided. The security attributes defined in the object-oriented model can be stored in the DFR documents for objects, and in a X.500 directory [CCITT92a] for subjects. Another standard of the X series, X.509 [CCITT92b], is used for secure authentication.

\subsection{The DFR standard and remote access to documents}

The DFR standard is well suited for our purposes as it is based on an object-oriented description of its document archive. The archive, called DFR store, is managed by one or more DFR servers, and accessed by end users via their DFR clients. A DFR document is comprised of two parts: the document content and a set of document attributes. A predefined list of attributes is available for DFR protocol support which can be extended by user-defined attributes, e.g. for specifying the document type for the application or, of course, for specifying security attributes. To obtain a structured hierarchy, DFR documents can be aggregated using the object type DFR group. The multiple appearance of documents in different groups without copying can be achieved using DFR references, which have the same content, but may have different attributes than the document they refer to. Figure 4 illustrates a DFR document store.

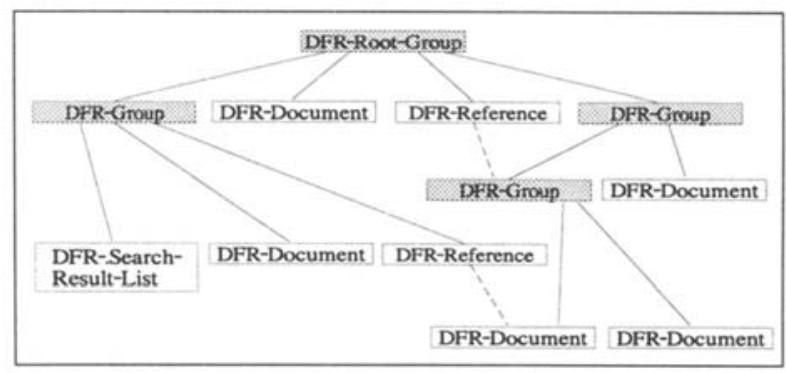

Figure 4: Structure of a DFR document store 


\subsection{The security services}

The security services are provided by dedicated security servers which interact with both the DFR client and the DFR server. In detail, we use an authentication server which identifies and authenticates a user"s identity. Successfully authenticated users obtain their privileges by the privilege server as a certified Privilege Attribute Certificate (PAC). Both obtaining authentication and privileges are based on the information which is derived from the X.500 directory [JuBu94]. On the DFR server side, service requests and PACs are checked by an authorization server. A security audit server is used to react to security relevant events of which it is notified by the other servers according to X.721 [ISO92].

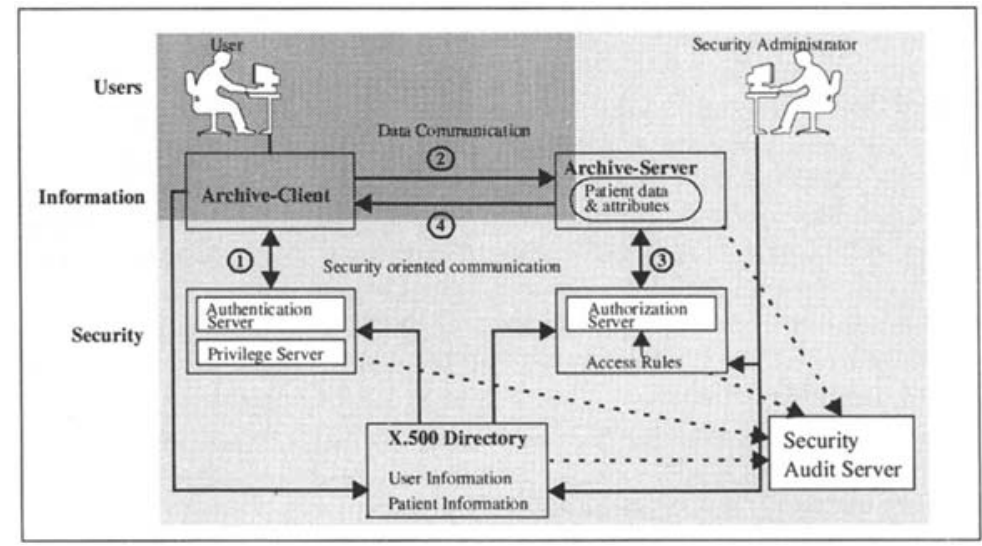

Figure 5: Cooperation of the Components

With this architecture, all characteristic features of user-oriented data security can be implemented. Following the user-oriented paradigm, the user may be able to define the security relevant actions or events as well as the reactions of the audit server, e.g. creating an entry in a $\log$ file or notifying a user.

\subsection{An application example}

A short example illustrates the cooperation of the different components: A physician wishes to request an X-ray diagnosis of one of his patients. His DFR client first sends the authentication data to the authentication server which compares these data with the data stored about the physician in the X.500 directory.

After successful authentication, the DFR client receives the appropriate PAC by the privilege server. This PAC is digitally signed by the trusted privilege server and thus cannot be modified without detection. The time of its validity can be defined so that it may be used several times during a session.

After successful authentication, the physician transfers to the radiologist a right to create a new diagnosis entry in the patient's file which is done by assigning a privilege attribute in the radiologist's X.500 entry. Additionally, an e-mail to the radiologist is sent containing the request and its medical details.

After taking the $\mathrm{X}$-ray exposure, the radiologist who got the new PAC containing the new access right creates a new diagnosis entry. The PAC is sent together with the DFR create 
request to the DFR server which gives both of them to its authorization server. The latter checks, using data it receives from the X.500 directory, if the radiologist is al lowed to issue the access. Since this is true, it will create the new document. The requesting physician will be notified automatically when this happens.

His read request is handled in a similar manner. His PAC containing the information at which ward he works is sent to the DFR server. As an implemented rule, the authorization server knows that a physician is allowed to read all patient data for the ward he belongs to. After having found the ward the patient belongs to, the authorization server applies this rule and allows the access.

\section{Summary}

We argued that centralized security systems are not able to satisfy the modern society's requirements on security and privacy. After a description of the social need for users specifying their requirements and the gap between requirements and solutions we introduced a new security approach called user-oriented data security which bases on an object-oriented model. User-oriented data security serves the users to let them specify all their security needs. This procedure allows for delegating the responsibility to them.

The description of use and its requirements of IT in a heal th care environment preceded the presentation of the technical architecture of our prototype which is based on international standards. An appropriate combination of security servers for authentication, privilegation, authorization, and audit together with the directory service serves as a technical basis in order to implement user oriented data security. A practical example of how the the components cooperate in a typical hospital process concluded the paper.

\section{References}

[BDSG91] Gesetz zur Fortentwicklung der Datenverarbeitung und des Datenschutzes. Bundesgesetzblatt 1990, Part 1.

[CCITT92a] Comité Consultatif International de Télephonique et Télégraphique: X series Recommendations. X.500: Data Communication Networks, The Directory. Geneva 1992.

[CCITT92b] Comité Consultatif International de Télephonique et Télégraphique: X series Recommendations. X.509: Data Communication Networks, The Directory, Authentication Framework. Geneva 1992.

[DaPr89] D. W. Davies and W.L. Price: Security for Computer Networks. 2nd ed., John Wiley and Sons, 1989

[DeFa91] Paula Lobato de Fario: A Survey of Legal Issues and Gaps in Legal Coverage in the EC. In: Commission of the European Communities (ed.): Data Protection and Confidentiality in Health Informatics. IOS Press, 1991.

[Denn82] Dorothy Denning: Cryptography and Data Security. Addison-Wesley 1982.

[ECMA88] ECMA TR/46: Security in Open Systems, A Security Framework. European Computer Manufacturers Association, Geneva 1988.

[ECMA89] ECMA-138: Security in Open Systems, Data Elements and Service Definitions. European Computer Manufacturers Association, Geneva 1988.

[Holz94] Gerard J. Holzmann: Data Communications: The first 2500 years. In: K. Brunnstein and E. Raubold (ed.): Proc. 13th IFIP World Computer Congress, Vol. 2. 
Elsevier 1994.
[ISO89] ISO/IEC International Standard 10165:Information Processing Systems - Open Systems Interconnection - Basic Reference Model, Part 2: Security Architecture. International Organization for Standardization, 1989.

[ISO91a] ISO/IEC International Standard 10166-1: Document Filing and Retrieval (DFR), Part 1: Abstract Service Definition and Procedures. ISO/IEC, 1991.

[ISO91b] ISO/IEC International Standard 10166-2: Document Filing and Retrieval (DFR), Part 2: Protocol Specification. ISO/IEC, 1991.

[ISO92] ISO/IEC International Standard 10165-2: Structure of Management Information: Definition of Management Information. ISO/IEC, 1992.

[ISO94] ISO Working Draft 11768: Information Security - Security Techniques - Evaluation Criteria for IT Security. International Organization for Standardization, 1994.

[JKP94] Marjan Jurecic, Ulrich Kohl, and Ernst Pelikan: SaferCom - ein Protoyp für Datenschutz in verteilten Klinikanwendungen. Datenschutz und Datensicherung $3 / 94$, vieweg.

[JuBu94] Marjan Jurecic and Herbert Bunz: Exchange of Patient Records - Prototype Implementation of a Security Attributes Service in X.500. Proc. 2nd ACM Conference of Computer and Communications Security, 1994.

[Lawr93] L.G. Lawrence: The Role of Roles. Computers \& Security 12 (1993)

[Lubi93] Astrid Lubinski: Ein Rollen-Normen-Modell für den konzeptionellen Entwurf von Sicherheitsanforderungen in Unternehmens-Informationssystemen. In: Gerhard Weck and Patrick Horster (ed.): Proc. Verläßliche Informationssysteme VIS '93, DuD Fachberichte, vieweg 1993

[Mart92] Raymond J. Martin: IBM Security Architecture: A Model for Securing Information Systems. IBM Corporation, 1992.

[NRC91] National Research Council: Computers at Risk: Safe Computing in the Information Age. National Academy Press, Washington, D.C. 1991.

[Rann93] Kai Rannenberg: Recent Development in Information Technology Security Evaluation - The Need for Evaluation Criteria for Multilateral Security. In: Richard Sizer at al. (ed.): Proc. IT Security and Control of IT in Society. Stockholm/St. Petersburg. IFIP Transactions A43, North-Holland 1993.

[Sand89] Ravi S. Sandhu: Transformation of Access Rights, Proc. IEEE Symposium on Research in Security and Privacy, IEEE Computer Society Press 1989

[Seel91] Hans-Jürgen Seelos: Informationssysteme und Datenschutz im Krankenhaus. vieweg 1991.

[Wäch94] Michael Wächter:Was ist und was soll Datenschutz? Datenschutz und Datensicherung 2, vieweg 1994.

[Wal192] Josef Wallmannsberger: The pragmatics of communicating with the computer: Towards a post-'fetishistic' theory of natural language processing. In: Peter Kaser, Josef Wallmannsberger (Ed.): Recht, Sprache und Elektronische Semiotik. Peter Lang, 1992. 